\title{
Helicobacter pylori in immigrants: a "foreign" bacterium?
}

\author{
Angelo Zullo $\cdot$ Francesca Cristofari • \\ Maria Consiglia Bragazzi · Cesare Hassan
}

Received: 8 September 2010/Accepted: 27 October 2010/Published online: 20 November 2010

(C) SIMI 2010

Helicobacter pylori infection is a worldwide spread disease with a definite morbidity and mortality. Indeed, the bacterium is the leading cause of both peptic ulcers and gastric tumors, including low-grade MALT-lymphoma and adenocarcinoma [1]. Although $H$. pylori prevalence is decreasing in developed countries, it remains very high in developing countries. Low socio-economic and educational levels, living in a family with a high number of siblings, and use of external water have been identified as risk factors for infection onset [2]. Moreover, in some countries, such as Chile and Peru, H. pylori has been isolated from municipal water, while it is absent in the developed countries [3]. Since the infection persists long-life, if not opportunely treated, infected patients who move from a developing country retain the infection also in the developed countries. There is evidence that $H$. pylori acquisition in developing countries occurs early in childhood, and, therefore, subjects in these geographic areas are at increased risk of developing gastroduodenal lesions despite young age. The recent study by Gatta et al. [4] shows that immigrant patients from East Europe who undergo upper endoscopy for dyspeptic symptoms have a significantly higher prevalence of peptic ulcer as compared to Italian patients (54 vs. 29\%), despite a lower median age (39 vs. 55 years). How can we interpret such a finding? As above mentioned, this could depend on the early acquisition of infection in these patients. Alternatively, immigrants may be infected with more virulent-i.e. cagA-positive [5]$H$. pylori strains more frequently than Italian patients.

\footnotetext{
A. Zullo $(\bowtie) \cdot$ F. Cristofari · M. C. Bragazzi · C. Hassan Gastroenterology and Digestive Endoscopy,

"Nuovo Regina Margherita" Hospital,

Via E. Morosini, 30, 00153 Rome, Italy

e-mail: zullo66@yahoo.it
}

However, a potential selection bias in patients enrollment in this study cannot be excluded. For example, due to cost for both endoscopy and histology, it is possible that immigrants will be convinced on the necessity for endoscopy only when either dyspeptic symptoms become more intense or persistent, or alarm symptoms occur, such as bleeding or persistent vomiting due to an ulcer complication. Unfortunately, the prevalence rate of alarm symptoms in neither immigrants nor Italians is not reported in the manuscript. Unexpectedly, peptic ulcer prevalence in this study would appear very high $(29 \%)$, even in the Italian dyspeptic patients. Indeed, in previous studies performed in the same geographic area, peptic ulcer prevalence is cited as $6.3-17 \%[6,7]$. Moreover, a prevalence rate as low as $2.7 \%$ on 11,148 consecutive upper endoscopy procedures is reported in a recent study performed in Rome [8]. In addition, in the study by Gatta et al. [4], a very similar prevalence of either duodenal (DU) or gastric ulcer (GU) is observed both in Italian (15.2 vs. 13.4\%) and in immigrant (29.3 vs. $25 \%$ ) patients. This would be in contrast with the higher prevalence of DU as compared to GU widely reported in literature (DU vs. GU = 3:1) [6]. As the Authors point out, the significance of all these findings remains unclear.

Another relevant finding in the study by Gatta et al. [4] is that H. pylori eradication rate following standard, 7-day triple therapy is disappointingly low. In detail, 1 of every 3 Italian, and 1 of every 2 immigrant patients remain infected despite therapy. As the authors suggest, the lower efficacy of eradication therapy in immigrants may depend on a higher antibiotic resistance. However, a recent study performed in Bulgaria shows a $15.4 \%$ primary clarithromycin resistance rate [9], a value very similar to the $16.9 \%$ quoted in Italy [10]. Moreover, the therapeutic regimen chosen by Gatta et al. [4] did not include metronidazole for which 
primary resistance rate is high in $H$. pylori strains isolated in the East population. Indeed, they administered amoxicillin for which primary resistance was very low both in Bulgaria (1.1\%) and in Italy [9, 10]. Therefore, it is likely that the lower eradication rate observed in immigrants depends on a low rate of compliance with therapy rather than on a high antibiotic resistance rate. Cost of drugs, troubles with the language in understanding the therapy regimen, and difficult familial circumstances may be the causes for concerns. On the other hand, a different distribution of CYP2C19 genotypes between the two patient groups is unlikely. Indeed all the enrolled patients were Caucasians, and, consequently, a high prevalence of rapid metabolizers of proton pump inhibitor in the enrolled immigrants causing a reduced efficacy of the eradication therapy is largely unlikely. In Orientals, there is a higher prevalence rate of poor metabolizers (15-23\%)-rather than rapid metabolizers - as compared to Western peoples (1-7\%) [11].

In conclusion, this is the first study showing that dyspeptic, $H$. pylori-positive immigrants living in our country should probably be managed differently from Italian patients. Whether the higher peptic ulcer prevalence and the lower eradication rate observed in these patients as compared to Italians actually depend on either a different genetic background between peoples or a "foreign" $H$. pylori remains to be ascertained.

Conflict of interest None.

\section{References}

1. Caselli M, Zullo A, Maconi G et al (2007) Cervia II Working Group Report 2006: guidelines on diagnosis and treatment of Helicobacter pylori infection in Italy. Dig Liver Dis 39:782-789
2. Kandulski A, Selgrad M, Malfertheiner P (2008) Helicobacter pylori infection: a clinical overview. Dig Liver Dis 40:619-626

3. Bellack NR, Koehoornl MW, Macnab YC, Morshed MG (2006) Conceptual model of water's role as a reservoir in Helicobacter pylori transmission: a review of the evidence. Epidemiol Infect 134:439-449

4. Gatta L, Di Mario F, Vaira D et al (2010) Helicobacter pylori eradication: are we really all equal? A controlled study in native and immigrant population. Intern Emerg Med doi:10.1007/ s11739-010-0443-0 [Epub ahead of print]

5. Douraghi M, Talebkhan Y, Zeraati H, Mohammadi M (2010) Cooperative genotyping for Helicobacter pylori virulence determinants strengthens the predictive value of gastric cancer risk assessment. Dig Liver Dis 42:662-663

6. Nervi G, Liatopoulou S, Cavallaro LG et al (2006) Does Helicobacter pylori infection eradication modify peptic ulcer prevalence? A 10 years' endoscopical survey. World J Gastroenterol 12:2398-2401

7. Di Mario F, Aragona G, Dal Bò N et al (2003) Use of bovine lactoferrin for Helicobacter pylori eradication. Dig Liver Dis 35:706-710

8. Sbrozzi-Vanni A, Zullo A, Di Giulio E et al (2010) Low prevalence of idiopathic peptic ulcer disease: an Italian endoscopic survey. Dig Liver Dis 42:773-776

9. Boyanova L (2009) Prevalence of multidrug-resistant Helicobacter pylori in Bulgaria. J Med Microbiol 58:930-935

10. Zullo A, Perna F, Hassan C et al (2007) Primary antibiotic resistance in Helicobacter pylori strains isolated in northern and central Italy. Aliment Pharmacol Ther 25:1429-1434

11. Chaudhry AS, Kochhar R, Kohli KK (2009) Importance of CYP2C19 genetic polymorphism in the eradication of Helicobacter pylori in north Indians. Ind J Med Res 130:437-443 